\title{
Reibungsgewinne. Identitäten im Inner[Außer]Halb
}

\author{
Angelika Frühwirth* und Ana Mijić**
}

\section{Zusammenfassung}

Wer bleiben möchte, hat sich anzupassen; wer ansprechen möchte, hat spezifischen Idealen zu entsprechen; wer leben möchte, hat zu funktionieren. Ausgehend von diesen, in den Werken dreier Wiener Autorinnen mit "Jugoslawien“-Bezug identifizierten "Gesetzen“ einer erstarrten Wirklichkeit, widmet sich dieser Beitrag der Frage, wie Selbstbilder in verdinglichten Systemen entworfen werden und wie viel Platz dabei individuellen Freiheiten noch vorbehalten bleibt. Der literaturwissenschaftlichen Analyse dieser Werke wird die hermeneutische Interpretation von Gesprächen mit Menschen mit Migrationserfahrung dialogisch gegenüber gestellt. Dabei zeigen sich wesentliche Analogien zwischen dem Umgang der RomanprotagonistInnen mit einer erstarrten Wirklichkeit und den in den Interviews auftauchenden Strategien zum Entwurf von diasporischen Identitäten: Assimilation, Überkompensation, Erstarrung oder Resignation lassen sich sowohl aus den Romanen als auch aus den Interviews rekonstruieren. Hier und dort manifestieren sich aber auch widerständige Momente. Durch die gezielte Erzeugung von Reibung bemächtigen sich die im Inner[Außer]Halb Positionierten wieder der Kontrolle über ihre eigene Identität.

Schlagwörter: Identität(en), Fremdheit/Entfremdung, Reibung/Konflikt

\section{Benefits of Friction. Inner[Out]Side Identities}

\section{Abstract}

Whoever wants to stay, has to adapt; whoever wants to approach someone, has to match specific ideals; whoever wants to live, has to function. Based on those claims of a rigidified reality identified in the novels of three Viennese authors with "Yugoslavian" backgrounds, this paper pursues the question of how self-images in such reified/rigid systems are forged and how much space for individual liberties still remains. The literary analysis of the novels is contrasted with a hermeneutical interpretation of interviews that have been conducted with individuals who have experienced migration. The contrasting juxtaposition shows that basic analogies can be identified between the ways the protagonists of the novels deal with rigidified realities and the strategies for shaping diasporic identities detected in the interviews: assimilation, overcompensation, rigidification or resignation can be identified in both the novels and the interviews. Occasionally, however, moments of resistance also become manifest in the material. By deliberately generating friction, the characters in the novels and interviewees positioned in the inner[out]side regain control over their own identity.

Keywords: identities, foreignness/alienation, friction/conflict

\footnotetext{
* Angelika Frühwirth. E-Mail: angelika.fruehwirth@gmx.at

** Ana Mijić, Hertha-Firnberg-Forschungsstelle (FWF) am Institut für Soziologie der Universität Wien. E-Mail: ana.mijic@ univie.ac.at

Die für unsere Analyse herangezogenen narrativen Interviews wurden im Sommersemester 2016 im Rahmen des Seminars „Hermeneutische Interpretation von Texten“ unter der Leitung von Ana Mijić am Institut für Soziologie der Universität Wien von Studierenden erhoben, transkribiert und z.T. auch analysiert. An dieser Stelle wollen wir uns sowohl bei den InterviewpartnerInnen als auch bei den SeminarteilnehmerInnen bedanken, dass sie uns das Material für eine weitere Verwendung zur Verfügung gestellt haben.
} 


\section{Einleitung}

„Reibungslos sollte die Kommunikation ablaufen, das war das Ideal, keine Reibung, keine Verluste“ (Dabić 2017: 36). So beschreibt die Protagonistin Nora in Mascha Dabićs 2017 erschienenem Roman Reibungsverluste die Erwartungen, die man an sie als Dolmetscherin in einem Therapiezentrum für traumatisierte Flüchtlinge stellt. Eindeutigkeit ist die Zielvorgabe. Ausdrücke, für die es im Deutschen kein Äquivalent gibt, lässt Nora deshalb beim Dolmetschen weg. Und Nuancen, die Missverständnisse erzeugen oder irritieren, macht sie unhörbar (Dabić 2017: 94, 119). Das Gesagte soll einorden- und damit kontrollierbar bleiben. Da jede Irritation Zweifel nach sich ziehen kann, gilt es diese zu vermeiden - keine Reibung, keine Verluste.

Reibungsverluste erzählt von Räumen, denen Reibung entzogen wurde, von Räumen der Kälte. Denn Reibungsverluste sind, so Nora, eigentlich „nichts anderes als Wärme, genau genommen die Umwandlung von Bewegungsenergie in Wärmeenergie, das wusste Nora noch aus dem Physikunterricht“ (Dabić 2017: 36). Die Vermeidung dieser „Wärme“ in einer Welt, in der Kommunikation und Begegnung ohne Reibung ablaufen sollen, kommt dem Verlust der Menschlichkeit gleich: „Im ersten Stock sah Nora manchmal Männer und Frauen mit nassen Haaren, in Schlafröcken oder Jogginganzügen, mit verschlossenen Gesichtern, Menschen, die aussahen, als sei ihnen das Warten auf den Asylbescheid in jede Faser des Körpers eingeschrieben, als habe ihre gesamte Existenz den Aggregatszustand des Wartens angenommen" (Dabić 2017: 27f.).

Mascha Dabić zeichnet eine Wirklichkeit, die Reibung vermeidet, um sich als „in sich geschlossenes, perfekt funktionierendes,System “" zu erhalten (Berger/ Luckmann 1969: 113), eine Wirklichkeit, die ihre Individuen - ob nun gewollt oder ungewollt - entmenschlicht und zu Rädchen degradiert.

1 In ihrer gemeinsamen Abhandlung Soziale Mobilität und persönliche Identität widmen sich Berger und Luckmann der Frage nach den Besonderheiten von Identität in der modernen Gesellschaft und stellen fest, dass einer „scheinbar schrankenlosen Autonomie" (1980: 150) im Privaten paradoxerweise ein „nahezu totaler Konformismus“ im Öffentlichen gegenübersteht. Denn in der Öffentlichkeit, so die Autoren, werde das Individuum gezwungen, „eng definierte Funktions-Rollen“ zu spielen und einzig als „Rädchen in der Maschinerie“ aufzutreten: „Dieses Paradox hat seine Wurzeln in einer totalen Verhaltens-Kontrolle durch primäre Institutionen, die verbunden ist mit Desinteresse an der Person.
In unserem Beitrag möchten wir der Frage nachgehen, wie Menschen, die sich möglicherweise nicht „reibungslos“ in etablierte, gesellschaftlich verfestigte Wirklichkeiten einfügen können/wollen/dürfen, in der Konfrontation mit diesen Wirklichkeiten ihre Selbstbilder entwickeln. Konzentrieren wollen wir uns dabei auf den sogenannten „Migrationshintergrund“ als eine von vielen möglichen Reibungsursachen. ${ }^{2}$ Im Wesentlichen wollen wir beleuchten, wie sich vor dem Hintergrund einer direkten oder indirekten Migrationserfahrung ${ }^{3}$ das Zusammenspiel von Individuum und Gesellschaft gestaltet, um abschließend auch darüber zu reflektieren, welche möglichen Reibungsgewinne die Begegnungen mit Unerwartetem, Neuem und Vielfalt (für die Gesellschaft und das Individuum) in sich tragen.

Zum Ausgangspunkt nehmen wir die Erkenntnisse, die unsere wissenschaftliche Zusammenarbeit hinsichtlich diasporischer Identitäten im Schreiben dreier Wiener Autorinnen mit „Jugoslawien“-Bezug (Sandra Gugić, Olja Alvir und Mascha Dabić) ergeben hat (Frühwirth/Mijić 2016). Diesen Erkenntnissen wollen wir die hermeneutische Interpretation (vgl. Oevermann et al. 1979; Oevermann 2002) von insgesamt vier narrativen Interviews dialogisch gegenüberstellen, die im vergangenen Jahr mit jungen Erwachsenen mit Bosnien-Bezug geführt wurden.

In unserer Arbeit treffen so die soziologische und literaturwissenschaftliche Perspektive mit dem Ziel aufeinander, die jeweilige Sichtweise auf die hier behandelte Thematik auf zuweiten. Die Resultate einer rekonstruktiven Interpretation von narrativen Interviews und die Ergebnisse der literaturwissenschaftlichen Untersuchung belletristischer Texte lassen sich evidenterweise nicht gleichsetzen. Denn ein literarischer

Verglichen mit anderen historischen Situationen führt dies zu einer Unter-Bestimmung der Identität. In einem Bereich des Sozialsystems ist das Individuum als Handelnder, ja als Verhaltensmaschine, funktionalisiert, während es in einem anderen völlig sich selbst überlassen ist und allein seine vermeintlich ,wesentliche Identität" zu entdecken hat" (Luckmann/Berger 1980: 150).

2 Weitere Ungleichheitsdimensionen (Klasse, Geschlecht etc.) wollen wir im Rahmen dieser Ausführungen nicht explizit berücksichtigen. Es gilt jedoch zumindest flankierend darauf hinzuweisen, dass eine Überkreuzung verschiedener Formen potenzieller Diskriminierung zu eigenständigen, auch identitätsspezifischen Diskriminierungserfahrungen führen kann (Winker/Degele 2010; Anthias 2002, 2001).

$3 \quad \mathrm{Zu}$ den identitätsspezifischen Besonderheiten einer indirekten Migrationserfahrung vgl. etwa Somerville 2008; Levitt 2009; Waters/Levitt 2002. 
Text bildet „nicht irgendein alltagspraktisches Handeln $\mathrm{ab}$, sondern stellt die methodisch kontrolliert erzeugte Objektivation eines künstlerischen Handelns dar" (Oevermann 1990: 246), so etwa der Soziologe Ulrich Oevermann. Selbst in literarischen Texten mit hohem autobiografischen Anteil sind soziale Gegebenheiten sowie individuelle Erfahrungen künstlerisch verarbeitet und reflektiert. In den hier behandelten Texten verhandeln die Autorinnen mit einem für gesellschaftliche Dynamiken empfindlichen Blick Prozesse der Identitätsbildung multidimensional und zeigen, wie Mechanismen der Verortung funktionieren oder scheitern können. Der Identifikationsfaktor für die Autorinnen selbst liegt dabei nicht im Erzählten (histoire), sondern vielmehr im Erzählenden (discours), d.h. im kreativen Akt des Schreibens bzw. im „Wie des Erzählens“. Daran lässt sich auch die Relevanz des Faktors Sprache (Ausdruck) und sprachlicher Konstruktion im Kontext der Identitätsbildung (hier im speziellen Fall der Autorinnen) festmachen. Literarische Imaginationen sind sich ihrer Fiktionalität bewusst und hegen keinen Anspruch auf Wirklichkeit, sehr wohl aber auf eine Wahrheit, die der behandelten Thematik innewohnt (Frühwirth/ Mijić 2016).

Neben der empirischen Ebene der narrativen Interviews und der literarischen Ebene der drei Romane müssen wir uns hier auch auf eine dritte, eine theoretische Ebene einlassen. Denn die aus der Theorie hervorgehenden beobachtungsleitenden Annahmen ermöglichen es uns, zumindest „(temporär) die Frage [zu klären], wie die Beschaffenheit sozialer Ordnung soziologisch zu verstehen und zu erforschen ist" (Kalthoff 2008: 12). Den theoretischen Hintergrund unserer Analyse bildet die Wissenssoziologie, wie sie von Peter L. Berger und Thomas Luckmann in Die Gesellschaftliche Konstruktion der Wirklichkeit entwickelt wurde. In der von ihnen herausgearbeiteten ,fundamentalen Dialektik des Sozialen' findet die für unsere Thematik elementare Frage nach der Verbindung von Individuum und Gesellschaft ihren systematischen Niederschlag. „Gesellschaft“ - so die Autoren - „ist ein menschliches Produkt. Gesellschaft ist eine objektive Wirklichkeit. Der Mensch ist ein gesellschaftliches Produkt“ (Berger/Luckmann 1969: 65). Durch soziales Handeln erschaffen, gerinnt Gesellschaft zu einer objektiven Wirklichkeit, die den Menschen auch unerbittlich gegenübertreten kann. Sie ist da; wir können sie ver-, aber nicht wegwünschen (Berger/Luckmann 1969: 64). Im Rahmen der Sozialisation wird diese objektive Wirklichkeit von den Menschen internalisiert und $\mathrm{zu}$ ihrer subjektiven Wirklichkeit, deren Schlüsselelement - so Berger und Luckmann - die Identität, ${ }^{4}$ das Selbstbild der Menschen ist. Diese Zusammenhänge werden im weiteren Verlauf unserer Ausführungen noch detaillierter dargelegt. Unsere Auseinandersetzung mit den Romanen von Gugić, Alvir und Dabić führte uns zu der Erkenntnis, dass die Autorinnen ihre Protagonistinnen schonungslos mit dem, was Berger und Luckmann als objektive Wirklichkeit bezeichnen, konfrontieren. Nicht nur das: Sie zeichnen eine verdinglichte Wirklichkeit. Verdinglichung wird in Die gesellschaftliche Konstruktion der Wirklichkeit als der äußerste Schritt der Objektivation betrachtet, ${ }^{5}$ es ist der Schritt, „durch den die objektivierte Welt ihre Begreifbarkeit als eines menschlichen Unterfangens verliert und als außermenschlich, als nicht humanisierbare starre Faktizität fixiert wird“ (Berger/Luckmann 1969: 96). Verdinglichung bedeutet also, „dass die Dialektik zwischen dem menschlichen Produzenten und seinen Produkten für das Bewußtsein verloren ist" (Berger/ Luckmann 1969: 95). Den Institutionen wird so ein ontologischer Status verliehen.

In einem ersten Schritt wird zunächst der literarische Ausgangspunkt mit seiner reflexiven Gesellschaftsperspektive im Zentrum der Betrachtung stehen. Ausgehend davon wollen wir in einem zweiten Schritt

4 Das Selbst ist, so Berger und Luckmann, in unmittelbarer Tradition zum symbolischen Interaktionismus und deren Vordenker (Cooley 1964; Mead 1934) „ein reflektiertreflektierendes Gebilde, das die Einstellungen, die andere ihm gegenüber haben und gehabt haben, spiegelt" (Berger/ Luckmann 1969: 142); sie verweisen aber auch darauf, dass es sich dabei um keinen „einseitige[n], mechanische[n] Prozeß“ handelt: „Er enthält vielmehr eine Dialektik zwischen Identifizierung durch Andere und Selbstidentifikation, zwischen objektiv zugewiesener und subjektiv angeeigneter Identität“ (Berger/Luckmann 1969: 142). Oder in anderen Worten: „Identität des Individuums bildet sich als eine Konstellation von Selbstverständnissen aus, die in sozialen Prozessen ihren Ursprung haben. Die Identität des Einzelnen ist ebenso sehr eine soziale Konstruktion wie eine individuelle Schöpfung; sie entsteht aus einer dialektischen Wechselwirkung zwischen beidem“ (Luckmann/ Berger 1980: 148).

5 Gemeinsam mit Stanley Pullberg formuliert Berger an anderer Stelle: „Unter Verdinglichung verstehen wir das Moment im Prozeß der Entfremdung, mit dem das Merkmal des Ding-Seins zum Maßstab der objektiven Realität wird. Als wirklich kann dann nur noch begriffen werden, was den Charakter des Dings hat" (Berger/Pullberg 1965: 102). Hier, wie auch in der gemeinsam mit Luckmann formulierten Wissenssoziologie, kommt es zu einer Übertragung des Begriffs der Entfremdung ,auf die immaterielle Produktion: die Wirklichkeit“ (Berger/Pullberg 1965: 101). 
die verdinglichten Systeme, so wie sie in den Romanen dargestellt werden, und die darin involvierten und einer unumgänglichen „Integrationsforderung“ unterliegenden, entmenschlichten Körper in den Blick nehmen. In einem dritten Schritt geht es um die Verortung der literarischen Figuren in einem „Dazwischen“ oder im Inner-[Außer-]Halb, wobei auch das zentral durch Homi Bhabha vertretene theoretische Konzept des In-Between aufgegriffen werden soll. Daran anschlieBend tauchen wir in das empirische Material ab: Anhand einer ausgewählten Interviewsequenz wollen wir der Frage nachgehen, inwieweit sich die gesellschaftlichen Verdinglichungen und die Verortung im "Dazwischen“auch in den Wirklichkeitsauffassungen unserer Interviewpartnerin niederschlagen und wie sie ggf. damit umgeht. Ausgehend von den Erkenntnissen dieser rekonstruktiven Analyse weiten wir dann wieder den Blick und versuchen, bislang identifizierte Bewältigungsstrategien aus allen vier Interviews dialogisch den typischen Reaktionsmustern unserer Romanfiguren gegenüberzustellen. Wie verhalten sich die ProtagonistInnen/InterviewpartnerInnen im Angesicht der an sie gestellten Erwartungen? Lassen sie sich einordnen, ordnen sie sich ein, werden sie unsichtbar oder leisten sie Widerstand?

\section{Literarischer Ausgangspunkt}

Das literarische Ausgangsmaterial unserer Untersuchung sind drei Romandebüts: Sandra Gugićs Astronauten (2015), Olja Alvirs Kein Meer (2015) und Mascha Dabićs Reibungsverluste (2017). Sie thematisieren auf sehr unterschiedliche Weise, wie sich die Verhärtung von Außen- wie Binnengrenzen auf die Gesellschaft und vor allem auf diejenigen auswirkt, die sich nicht unmittelbar und unbemerkt in vorhandene Schemata und Strukturen einfügen können und/oder wollen.

Alle drei Autorinnen wurden noch zur Zeit des sozialistischen Jugoslawien geboren. Allerdings mit dem Unterschied, dass Olja Alvir (geb. 1989) und Mascha Dabić (geb. 1981) nach Ausbruch des Krieges mit ihren Familien nach Wien geflohen sind, während Sandra Gugić (geb. 1976) als Kind eines sogenannten GastarbeiterInnen-Paares in Wien zur Welt gekommen und aufgewachsen ist. Ihre Eltern stammen beide aus dem heutigen Serbien.

Während sich Mascha Dabić in Reibungsverluste, wie oben kurz skizziert, schwerpunktmäßig mit Asylpolitik und den Auswirkungen nationalstaatlicher „Grenzsicherung“ auseinandersetzt, beschäftigen sich
Sandra Gugić und Olja Alvir mit weniger sichtbaren Grenzen, von denen die gesellschaftliche Wirklichkeit durchsetzt ist. Sandra Gugićs Astronauten erzählt von sechs Charakteren, die an einem ,Nicht-Ort' (Augé 1992), in einer namenlosen Stadt, gestrandet sind. Der Zufall entscheidet genauso über Lebensgeschichten wie auch Freundschaften und Familie. Parameter von Herkunft, Tradition und Genealogie sind verloren, doch dadurch lange nicht alle Zwänge aus dem Leben geräumt. Die scheinbare Schwerelosigkeit verspricht statt Freiheit Gefangenschaft. Denn Gugićs Astronauten bewegen sich in einer Welt, die ihnen den Weg zu sich selbst verwehrt. Sie können sich in der Welt, die sie umgibt, nicht wiederfinden und so auch nicht Fuß fassen. Objektive und subjektive Wirklichkeit und damit auch Identität können nicht in Einklang miteinander gebracht werden.

In Olja Alvirs Kein Meer tastet die Studentin Lara die Grenzen ihrer durch Krieg und Migration (mit-) geprägten Identität und Lebenswelt ab. Dabei geraten ihr nicht nur Widersprüche der eigenen (Familien-) Geschichte, sondern auch gesellschaftspolitische und sehr körperbezogene Themen unter die Finger. Mit den Fragen, wer oder was ihrer Lebenswelt angehört und wovon sie sich abgrenzen darf, macht Lara nationalistisch, sozial und körperlich etablierte Kriterien von Zugehörigkeit in doppeltem Sinne angreifbar: Sie erfasst sie mit der Haut - ihrer Reibungsfläche mit der Welt - und stellt sie zugleich kritisch zur Debatte.

\section{Erstarrte Wirklichkeiten \& entmenschlichte Körper}

Trotz der Fokussierung auf sehr unterschiedliche Handlungen beschäftigen sich die drei ausgewählten Romane im Kern mit der Konfrontation mit Widersprüchlichem, Irritierendem. Oder anders gesagt: mit der Begegnung von „repulsiven Kräften“, denn bemerkenswerterweise bedienen sich alle drei Autorinnen immer wieder physikalischer - vereinzelt auch chemischer - Begriffe und Erklärungen. So als könnten die Gesetze der Naturwissenschaften ohne Weiteres auf soziale Geschehnisse übertragen werden. Beispielsweise überzeichnet Olja Alvir in Kein Meer das sterile Paradigma der Schönheitsindustrie, indem sie diverse Ausscheidungsvorgänge des menschlichen Körpers einer Art physikalischen Normierung unterwirft: Die Oberflächenspannung bestimme den Moment, da sich beim Pickelausdrücken ein Blutstropfen löse (Alvir 2015: 129). Adhäsionskraft und Fließgeschwin- 
digkeit seien wiederum für die Fallkurve des Urinstrahls ausschlaggebend (vgl. ebd., 98). Mascha Dabić spricht in Reibungsverluste vom „Aggregatszustand des Wartens“, in den AsylwerberInnen vom System versetzt würden (Dabić 2017: 27). Erstaunliche Überschneidungen hinsichtlich ihrer physikalischen Argumentation weisen alle drei Werke dort auf, wo soziale Interaktion beschrieben wird. Diese beschränkt sich in Astronauten und Kein Meer auf die von Zufall und Wahrscheinlichkeit gesteuerte Begegnung von Molekülen (Gugić 2015: 132) bzw. Atomen (Alvir 2015: 138), aus denen die Haut zusammengesetzt sei. Alvir kommt dabei zu dem Schluss, dass „Menschen [...] einander gar nicht berühren [können]. Die Atome, aus denen zum Beispiel Haut zusammengesetzt ist, kommen nie miteinander in Kontakt, weil sie jeweils von Elektronen umgeben sind. Und diese Elektronen sind nicht etwa in Schalen oder auf fixen Bahnen um die Atomkerne unterwegs, sondern befinden sich in einer sogenannten Aufenthaltswahrscheinlichkeit um den Atomkern herum. Man spricht von einer Elektronenwolke, die den potenziellen Aufenthaltsraum rund um den Atomkern umfasst und je nach Ort und Energieniveau höhere oder niedrigere Aufenthaltswahrscheinlichkeiten aufweist" (Alvir 2015: 138). Als ähnlich unwahrscheinlich beschreibt Mascha Dabić eine wahrhaftige Begegnung mit dem menschlichen Gegenüber, indem sie ihre Protagonistin in Räumen der Kälte verortet. Auf die Vermeidung von Reibung ausgerichtete Systeme würden das Aufkommen von Wärme in zwischenmenschlichen Begegnungen verhindern (Dabić 2017: 36).

Tatsächlich spiegelt sich in diesen Vergleichen von Sozialem mit Physikalischem das Gesellschaftsbild, das die Autorinnen in ihren Romanen zeichnen: In allen drei Texten sind die Individuen mit einem Außen konfrontiert, welches ihnen zwar in zergliederter Ausformung, aber dennoch unerbittlich starr gegenübersteht. Vom Menschen geschaffene Normen scheinen „mit der Natur zu verschmelzen“ (Berger/Luckmann, 1969: 97) und werden, berechenbaren Naturgesetzen gleich, zur Richtschnur darüber, wie Körper, Lebensentwürfe, Karrieren etc. auszusehen haben: Oberflächen sollen fest, „abwischbar“ (Gugić 2015: 50), „glatt und konstant [sein]“ (Alvir 2015: 138), denn nur glatte Oberflächen schweigen (Frühwirth/Mijić 2017: 40; Gugić 2015: 27). „[D]a stören alle Unregelmäßigkeiten“ (Alvir 2015: 138). Biografien haben „geradlinig“ zu verlaufen (Gugić 2015: 64), denn: „Man muss sich in geraden Linien vorwärts bewegen“ (Gugić 2015: 61). Und auch die „launische“, die „sprunghafte“ Erinnerung, die „von einem
Zimmer in das andere [läuft]" (Gugić 2015: 35), muss gezähmt werden, sie soll - wie jede Fluchtgeschichte - chronologisch rekonstruierbar sein (Dabić 2017: 118). Vorhersehbarkeit, Eindeutigkeit, Einordenbarkeit ermöglichen Kontrolle. Und nur durch Kontrolle kann das perfekt funktionierende System aufrechterhalten, das drohende Chaos in Schach gehalten werden.

"Jede gesellschaftliche Wirklichkeit ist gefährdet und jede Gesellschaft eine Konstruktion am Rande des Chaos" (Berger/Luckmann 1969: 111), formulieren Berger und Luckmann. Obwohl ein Produkt des menschlichen Tuns, stehen - wie bereits eingangs formuliert - die gesellschaftlichen Realitäten dem Menschen als „objektive Faktizität unabweisbar gegenüber. Sie sind da, außerhalb der Person und beharren in ihrer Wirklichkeit, ob wir sie leiden mögen oder nicht“ (Berger/Luckmann 1969: 64). Im Zuge der Sozialisation wird diese objektive Wirklichkeit internalisiert und Teil der subjektiven Wirklichkeit des Menschen. Als gesellschaftlich „perfektes“ Individuum ist jenes zu betrachten, bei welchem eine vollkommene Symmetrie zwischen objektiver und subjektiver Wirklichkeit vorliegt (Berger/Luckmann 1969: 175). Ein Individuum, das will, was es soll, das sich "reibungslos" in die gesellschaftlichen Wirklichkeitsvorstellungen einzugliedern, zu integrieren vermag: „Was ,außen` wirklich ist, entspricht dem, was ,innen' wirklich ist" (Berger/ Luckmann 1969: 144). Je weniger Konflikt, desto ungestörter die Reproduktion gesellschaftlicher Wirklichkeit; „keine Reibung, keine Verluste“ (Dabić 2017: 36). All jenes, das sich nicht einordnen oder "festnageln“ lässt (Gugić zieht immer wieder das Bild des gekreuzigten Jesus heran), wird als Bedrohung wahrgenommen und stellt für eine erstarrte Wirklichkeit tatsächlich auch eine Bedrohung dar. Denn das Bewegliche, das nicht Festgeklebte, oder das nur lose Verbundene (z.B. die AstronautInnen) macht auf Probleme, auf Leerstellen aufmerksam.

Als äußerste Reaktion auf die Erstarrung im Außen taucht in Gugićs Astronauten auch der Freitod, d.h. also die Erstarrung des menschlichen Körpers, auf. Der Stillstand der Welt im Außen führt zum Atemstillstand. Dies wird in der von Gugić beschriebenen Szenerie des Selbstmords bildsprachlich verdeutlicht: Der gehängte Körper scheint mit der Kulisse seiner starren „Lebenswelt“ zu verschmelzen: Hinter dem, dessen Füße „nur eine Handbreit über dem Boden [schweben], als wäre er dabei, eine Übung in Schwerelosigkeit zu absolvieren“" (Gugić 2015: 149), hängt ein Bild an der Wand, das an eine Mondlandschaft erinnert. Bei näherem Hinse- 
hen ist darin ein Puzzle zu erkennen, dessen Teile festgeklebt sind (Gugić 2015: 149): Das Spiel ist angehalten, zu Ende. Oder, wenn man so will: Endspiel, um Samuel Becketts Theaterstück zu erwähnen, dem Gugić eines der Mottos ihres Romans entnommen hat. Die Einzelteile des Puzzles, ursprünglich frei beweglich, sind $\mathrm{zu}$ einem Gesamtbild erstarrt, aus dem jegliches Leben gewichen ist.

Der Frage nach der eigenen Identität, die sich die Charaktere in den drei Romanen stellen, begegnen die Systeme, indem sie Identität zur Gewohnheit, zum Gesetz, erklären: Identität hat (be-)steuer- und überprüfbar zu sein. Gleich einer digitalen Filmprojektion zieht das Leben an den ProtagonistInnen in Gugićs Astronauten vorbei: „Verschwimmende Nullen und Einsen. Einschlafen und aufwachen“"(Gugić 2015: 89). Ähnlich getaktet verläuft Noras Arbeitsalltag in Dabićs Reibungsverluste: Jedem Tun wird ein Wort vorangestellt: hetzen, ankommen, übersetzen, dolmetschen, entspannen, pausieren. Nichts wird einem potenziellen Missverstehen überlassen. Ihr Tag wird in einzelne Etappen geteilt und damit „chronologisch rekonstruierbar" (Dabić 2017: 118) - so wie es Flucht- und Folterberichte zu sein haben. Passgenau sollen sie sein, die Bausteine einer Flucht; und genauso passgenau auch deren sprachliche Übertragung ins Deutsche. Wie ein Computer soll Nora sie Wort für Wort hinübertragen in die andere Sprache: lückenlos, ohne Leerstellen, ohne Fehler. Entseelt. „Eine verdinglichte Welt ist per definitionem eine enthumanisierte Welt“, so auch Berger und Luckmann (Berger/Luckmann 1969: 95).

Vielleicht sind die Menschen „nur Moleküle, die sich irgendwo neu zusammensetzen“ und „jedes Mal in einem anderen Universum [...] am falschen Ort zur falschen Zeit" wiederaufwachen (Gugić 2015: 132). Oder „eine Menge an Wahrscheinlichkeiten“ (Alvir 2015: 138), die aufeinandertreffen, aber niemals wirklich in Beziehung treten können. Vielleicht sind sie Roboter - ferngesteuert wie Gugićs Charaktere. Anweisungen erhalten sie über den Lautsprecher in der Bahn „Achten Sie auf Ihre Tasche, Geben Sie acht auf den Signalton, Steigen Sie nicht mehr ein“" (Gugić 2015: 115f) -, vom Familienrat oder „dem Gesetz“. So zeichnet z.B. einer der AstronautInnen seine Mutter als Exekutivorgan der vom Vater veranlassten Abschiebung in die „Heimat":

„Die verheulten Augen meiner Mutter beim Abschied, die ihre Standardsätze wiederholt hat, wie sich alles wiederholt, ist besser so, besser für die Familie, wir lieben dich, verstehst $d u$, die Familie liebt dich, meine Mutter hat die
Wunde an meinem Kopf genäht, fünf Stiche, das kann sie im Schlaf, die Krankenschwester aus der Hölle, aber da ist nichts zu reparieren, irreparabel, Mutter, die wiederholt, wiederkäut, ist besser für dich, besserbesserbesser. Küss deine Mutter, hat sie gesagt. Mama, du Sau, zeig mir, dass du keine Maschine bist" (Gugić 2015: 164).

Die AutorInnen wenden sich gegen die Verdinglichung und gegen die Enthumanisierung, indem sie schreiben, indem sie ihre ProtagonistInnen als ZweiflerInnen konzipieren, die aufgrund ihres Zweifelns auch leiden, und indem sie blinde Flecken der Systeme benennen, über die die „systemeigene“ Unmenschlichkeit überhaupt erst thematisierbar wird. Nun kann man darüber spekulieren, inwiefern die Biografien der Autorinnen bedeutsam für ihr künstlerisches Schaffen sind, doch eines ist evident: Ihren ProtagonistInnen - den AstronautInnen, der Dolmetscherin sowie der Schönheitsexpertin und Familiendetektivin - ermöglichen sie das Zweifeln dadurch, dass sie sie in einem „Dazwischen“ - in einem Innen, das auch gleichzeitig ein Außen ist - positionieren.

\section{Verortung im Inner-[Außer-]Halb - Theoretische Annahmen und literarische Verarbeitung}

In allen drei genannten Romanen wird die Suche nach Identität als Folge von ,Entwurzelung thematisiert. Diese subjektiv empfundene Entwurzelung ist die Konsequenz einer direkten oder indirekten Migrationserfahrung, grundlegend aber auch das Ergebnis der Flucht vor sozialen Erwartungen und starren Identitätsbildern. Die ProtagonistInnen werden von den Autorinnen in offene Räume gesetzt, in denen es keine vorgefertigten Antworten auf die mehr oder weniger existenziellen (und oft auch vorgefertigten) gesellschaftlichen Fragen gibt. Die Romane sind allein schon geografisch in solchen spaces in-between angesiedelt. Gugićs AstronautInnen leben in einer Stadt ohne Namen und ohne Geschichte. Lara, die Protagonistin in Alvirs Kein Meer, bewegt sich zwischen Österreich, Kroatien und Bosnien. Und die Welt der Dolmetscherin Nora spannt sich zwischen St. Petersburg, Wien und den Schauplätzen von Flucht und Folter auf, von denen ihre KlientInnen berichten.

Die Frage nach der Zugehörigkeit lässt sich hier nicht darauf beschränken, welcher Gruppe oder gesellschaftlichen Kategorie ein Individuum angehört - d.h. es geht nicht allein um die gesellschaftliche Verortung. Sie beinhaltet darüber hinaus auch immer die Frage danach, was zu dem Individuum gehört und was nicht, 
d.h. also die Auslotung der eigenen Grenzen (körperlich, biografisch etc.), die sich mit der Frage „Wie weit reicht mein Sein?" fassen ließe. In Kein Meer lotet Alvir diese Frage beispielsweise anhand einer unmittelbar körperlichen Erfahrung aus: der kosmetischen Haarentfernung (Alvir 2015: 37). Nora, die Dolmetscherin, fragt sich, inwieweit ihr Kopf ein Sammelbecken für alles nicht Vermittelbare darstellt (Dabić 2017: 94, 119). Doch insbesondere in Gugićs Astronauten drängt sich das „Dazwischen“ in Gestalt einer „Leerstelle“ bzw. der „Haltlosigkeit" auf: Alen, einer ihrer AstronautInnen, überarbeitet etwa seit Jahren ein und dasselbe Romanmanuskript, indem er es immer wieder übersetzt: Aus dem Deutschen in seine Muttersprache und aus seiner Muttersprache ins Deutsche: „Das Hin-undHer-Übersetzen ist eine Manie geworden, weil er immer Fehler findet, Ungereimtheiten, in jeder Sprache andere" (Gugić 2015: 13). Er oder besser gesagt: das, was er zu sagen hat, ist zwischen den Sprachen verloren gegangen. Übrig geblieben zu sein scheint Substanzlosigkeit: „Ich entdecke ein Leerzeichen zu viel zwischen trotz und allem“, so Alen, „eine Leerstelle, ein Wurmloch, das die Erinnerung und den Raum, in dem ich mich befinde, zwischen diese beiden Worte saugt. Alles könnte darin verschwinden. Der Cursor ist ein Ausrufungszeichen, wirft schwarze Buchstaben auf die weiße Fläche und läuft rückwärts, verschluckt alle wieder, pulsiert im Atemrhythmus auf dem weißen Bildschirm" (Gugić 2015: 30). Das Nichtvorhandensein der Plausibilität von vorbestimmten Realitätskonzepten, d.h. das Fehlen von Plausibilitätsstrukturen und damit auch von selbstverständlichen Verbindungen zur Wirklichkeit, zwingt die ProtagonistInnen dazu, neue Antworten zu finden auf die wiederkehrenden Fragen: „Wer bin ich?" oder: „Wer möchte ich sein?"

Wie auch theoretische Texte nahe legen, birgt diese Offenheit Risiken und Chancen gleichermaßen (vgl. etwa Bhabha 2000; Park 1950; Park 1928; Said 2002; Schütz 1972). In seiner wissenssoziologischen Auseinandersetzung mit dem Typus des Fremden schreibt etwa Alfred Schütz (dessen Sozialphänomenologie ein zentrales Fundament der Wissenssoziologie von Berger und Luckmann ist), dass die „Zivilisations- und Kulturmuster" - also verfestigte kulturelle Deutungsmuster - für Hinzukommende oder Hinaustretende, „kein Schutz, sondern ein Feld des Abenteuers, keine Selbstverständlichkeit, sondern ein fragwürdiges Untersuchungsthema, kein Mittel um problematische Situationen zu analysieren, sondern eine problematische Situation selbst [sind]" (Schütz 1972: 67).
Gleichzeitig weist er aber auch darauf hin, dass die Positionierung im Außerhalb von sozialen Zwängen befreien und zur kritischen Distanzierung und intensiveren Reflexion befähigen kann: ${ }^{6}$ Idealtypisch akzentuiert, formuliert Robert E. Park in seiner Abhandlung über den marginal man - den Grenzgänger: „Inevitably [s/]he becomes, relatively to [her/]his cultural milieu, the individual with the wider horizon, the keener intelligence, the more detached and rational viewpoint. The marginal man is always relatively the more civilized human being" (Park 1950: 375f.). Es ist vor allem diese positive Konnotation des Dazwischenseins, die in den postkolonialen Literaturwissenschaften von Homi K. Bhabha hervorgehoben wird. Das „Dazwischen“ wird als "dritter Raum“ verstanden, der eine objektive Wahrnehmungsperspektive privilegiert: „Im Bereich des darüber Hinauszugehenden zu sein heißt also, [...] einen Zwischenraum zu bewohnen. Aber im ,Darüber Hinaus' zu wohnen heißt auch [...] an einer re-visionären Zeit teilzuhaben, an einer Rückkehr zur Gegenwart, um unsere kulturelle Gleichzeitigkeit neu zu beschreiben; [...] die Zukunft auf der uns zugewandten Seite zu berühren. In diesem Sinne wird also der Zwischenraum des ,Darüber Hinaus' zu einem Raum der Intervention im Hier und Jetzt" (Bhabha 2000: 10).?

Auch wenn wir uns nicht darauf festlegen wollen, inwiefern das Schreiben der Autorinnen durch ihre Herkunft mitgeprägt wurde, ist es unserer Ansicht nach aber in jedem Fall als eine solche „Intervention im Hier und Jetzt" zu begreifen. Sie schreiben nicht nur von Reibungen, ihr Schreiben verursacht auch Reibung. Eine Reibung, die unseres Erachtens für eine Gesellschaft, die nicht erstarren will, von herausragender Bedeutung ist. Darauf wollen wir später nochmals eingehen.

6 In der klassischen Soziologie über das Fremde wird auf die „Objektivität“ des/der Fremden oder des/der Beweglichen hingewiesen. Nicht in den Wertekanon der Ingroup sozialisiert, erlebt die/der Fremde „das Naheverhältnis wie aus der Vogelperspektive“ (Simmel 1992: 767). Er/Sie muss hinterfragen, um zu verstehen (vgl. Schütz 1972: 67f.), dies wird typischerweise als „Zweifelhafte Loyalität“ (Schütz 1972: 68) ausgelegt.

7 Über die unterschiedlichen Erscheinungsformen des Dazwischenseis reflektiert z.B. Said in seiner Abhandlung über das Exil. So schreibt er etwa: „Paris may be a capital famous for cosmopolitan exiles, but it is also a city where unknown men and women have spent years of miserable loneliness [...] what do these experiences [of war and flight] add up to? Are they not manifestly and almost by design irrecoverable?" (Said 2002: 139). 
Wir haben uns hier nun aber die Frage gestellt, ob und wie sich direkte oder indirekte Migrationserfahrungen in den Selbst- und Wirklichkeitsauffassungen von Menschen niederschlagen, die weder künstlerisch schaffen, die also ihre individuellen Erfahrungen nicht künstlerisch verarbeiten und reflektieren - so wie die Autorinnen -, noch künstlerisch erschaffen wurden wie deren ProtagonistInnen. Um diese Frage zu beantworten, haben wir die Transkriptionen von narrativen Interviews, die im Frühjahr 2016 mit jungen Erwachsenen mit Bosnienbezug erhoben wurden, einer rekonstruktiven Analyse unterzogen. Narrative Interviews zeichnen sich durch einen offenen und erzählgenerierenden Interviewstil aus, der es den Interviewten d.h. den jeweiligen GesprächspartnerInnen - erlaubt, eigene Relevanzen zu setzen, mit der Erwartung, dass in solchen Erzählungen Orientierungs- und Deutungsmuster der SprecherInnen zum Ausdruck kommen, die dann im Rahmen der Analyse rekonstruiert werden (Loch/Rosenthal 2002). ${ }^{8}$ Eine ausführliche Darstellung der rekonstruktiven Analyse des gesamten Datenmaterials würde den Rahmen der Ausführungen sprengen. Aus diesem Grund haben wir uns dafür entschieden, die Interpretation einer ausgewählten Sequenz aus einem als Ausgangsprotokoll gesetzten Interview etwas detaillierter wiederzugeben und im Anschluss daran die zentralen Erkenntnisse unserer bisherigen Analyse - sowohl der literarischen Werke als auch aller narrativer Interviews - systematisiert und zusammenfassend darzustellen.

\section{5. „Zwischen zwei, drei Stühlen ...” - Alltagsweltli- che Perspektiven auf das Dazwischen-sein}

IA: (...) „was a interessant wäre, $i$ hab ähm bis zu meinem dreizehnten Joahr mit einem Akzent gesprochen, den hab i mir dann in der höheren Schule abtrainiert, also eben doch gewollt, mehr gewollt als ungewollt, ähm ja und

8 Ein narratives Interview beginnt mit einer erzählgenerierenden Einstiegsfrage. Im vorliegenden Fall wurden die InterviewpartnerInnen gebeten, ihre Lebensgeschichte zu erzählen (Loch/Rosenthal 2002). Dabei werden die Interviewees nicht unterbrochen. Erst im Anschluss an ihre Einstiegserzählung folgt zunächst ein immanenter Nachfrageteil, in dem versucht wird, Erzählungen zu noch nicht explizierten Themen (z.B. ausgelassene Lebensabschnitte) oder zu noch wenig ausgeführten Themen zu generieren. Ein exmanenter Nachfrageteil ermöglicht es den Interviewenden schließlich noch potenziell forschungsrelevante Fragen zu stellen. Im Rahmen der hier verwendeten Interviews wurden keine exmanenten Fragen gestellt. irgendwie äh naja man muss sagen i bin so zwischen zwei, drei Stühlen aufgewachsen, also kulturelle Einflüsse (2) am Stärksten merk $i$ es eher so am Essen die Tradition, ähm und da bin i sehr froh, weil $i$ kann mir aus all meinen Heimaten äh Sachen rausnehmen äh von denen i profitier. Naja, sprachlich eher weniger eher kulturell, also für mi persönlich (3)

F: Und hast du noch immer das Gefühl, dass Du zwischen zwei, drei Stühlen ...

IA: ... genau immer noch und i profitier immer noch sehr viel davon (3) es is allein die Art zu essen oder die Art zu leben, hab i oft des Gefühl ganz ne andere, weil man kann sich irgendwie, also es sind so Kanäle, die man entweder trennen kann oder a net und man kann sich eben ein bisschen aussuchen, wie man grad möchte, manchmal nimmt man sich die Vorteile aus allen Kulturen raus und des kann $i$ zum Beispiel, des find i sehr schön (2) ja eben diesen Körperkontakt, aber dann gleichzeitig mit irgendeiner Professionalität, die $i$ halt mit Österreich eher verbind, des kann man alles kombinieren oder auch nicht, aber $i$ hab halt das Gefühl, $i$ hab die Freiheit daraus $z u$ wählen, ja und natürlich a so Sachen äh wie Musik, Sprache an sich, da hat man a sehr viel breiteres Spektrum, wenn man, ähm, ja wenn man einfach so in verschiedenen Welten halt irgendwie aufgewachsen ist (2). (Interview A, Zeile $83 \mathrm{ff}$.)

Das (theoretisch angenommene, sich aus den literarischen Werken herauskristallisierte) „Dazwischen“ wird in diesem Gespräch geradezu paradigmatisch zum Ausdruck gebracht: Die Rednerin positioniert sich „zwischen zwei, drei Stühlen“. Die typischerweise problematische Positionierung zwischen zwei oder mehreren Stühlen, die mit der Formulierung „man muss sagen, i bin“ auch angedeutet wird, wird hier positiv umgedeutet - „i profitier davon“ - und dadurch entproblematisiert.

Zunächst einmal bleibt offen, wie dieser Profit, der Mehrwert, zustande kommt. Grundsätzlich sind zwei Wege denkbar: Entweder es wird im „Dazwischen“ ein hybrider Raum aufgespannt, in dem sich, im Zusammenspiel der bestehenden Kräfte (in der Reibung), etwas Neues entwickelt. Oder: Das Dazwischen stellt nur einen Ausgangspunkt dar, von dem aus man sich je nach Situation oder Bedarf - der einen oder anderen Seite angleicht.

Nun werden aber die unterschiedlichen kulturellen Einflüsse in dieser Sequenz als in sich abgeschlossene, unveränderbare Bereiche aufgefasst („verschiedenen Welten“, ,allein die Art zu leben' ist ganz eine andere), als ,Behälter, aus denen man sich, einer Konsumlo- 
gik folgend, bedienen kann. In den Ausführungen der Gesprächspartnerin manifestiert sich damit recht deutlich eine statische oder monolithische Kulturauffassung, die jeder Annahme von Hybridität entgegen steht.

Die Rede von Kanälen, die ,getrennt werden können oder nicht', verstärkt die zweite Lesart. Dass ein Abkappen von der einen oder anderen Welt als grundsätzlich möglich betrachtet wird, lässt sich nicht oder nur schwer in Einklang bringen mit der Vorstellung eines fließenden Zwischenraums. Es kann also plausibel davon ausgegangen werden, dass sich die Sprecherin je nach Situation auf dem einen oder dem anderen Stuhl platziert.

Auffällig ist dabei, dass die Interviewpartnerin bei der Beschreibung der „Heimaten“, ihrer kulturellen Einflüsse, scheinbar auf Stereotype zurückgreift („eben dieser Körperkontakt, aber dann gleichzeitig mit irgendeiner Professionalität, die i halt mit Österreich eher verbind“). Im weiteren Verlauf des Interviews zeigt sich dann, dass sie sich selbst zwischen diesen Stereotypen platziert. $^{9}$ Stereotypisch werden in der Regel aber nur „Objekte“ beschrieben, zu denen man keinen unmittelbaren Zugang hat, bei denen man über kein intimes Wissen verfügt. ${ }^{10}$ Hier drängte sich uns die Frage auf, ob die Stereotypisierung der eigenen Person darauf zurückzuführen wäre, dass Etikettierungen der Umwelt, dass Fremdzuschreibungen übernommen werden und eigene unhinterfragte und selbstverständliche Verbindungen zur Wirklichkeit letztlich fehlen. Dieser These werden wir sowohl theoretisch als auch am Material noch weiter nachgehen müssen. Bemerkenswert ist darüber hinaus, dass die Sprecherin immer wieder zum Ausdruck bringt, dass sie aus freien Stücken handelt: man kann die Kanäle trennen oder nicht, so wie sie sich den Akzent „mehr gewollt als ungewollt" abtrainiert hat, sie hat(te) die Freiheit zu wählen. „Mehr gewollt, als ungewollt“ bedeutet jedoch, dass es auch ungewollt geschah. Und: „man kann sich eben ein bisschen aussuchen, wie man gerade

9 „Menschen leben so in den Tag rein [...] i bin vielleicht ah zurückhaltender vom Temperament ... und im Österreichvergleich wahrscheinlich dann wieder temperamentvoller aber unten net, da bin i wahrscheinlich so höflich, zurückhaltender ja wie sie das halt viel mehr ausleben“ (Interview A, Zeile 702f.).

10 Alfred Schütz und Thomas Luckmann schreiben etwa: „Je anonymer der Typus, vermittels dessen ein Zeitgenosse erfahren wird, um so stärker ist der Sinnzusammenhang, der dem anderen untergeschoben wird, objektiviert" (Schütz/Luckmann 2003: 124). möchte“, bedeutet auch, dass die Wahlmöglichkeit eingeschränkt ist; die Entscheidung obliegt nicht nur dem eigenen freien Willen. Handelt es sich hier letztlich um eine erzwungene Anpassung an die eine oder andere objektive Wirklichkeit? Die Sprecherin präsentiert sich im Verlauf des Interviews also immer wieder als selbstbestimmt und aktiv handelnd - und doch wird diese Selbstdarstellung durch die Erzählung selbst immer wieder konterkariert, wie anhand der folgenden Sequenz exemplarisch gezeigt werden kann:

„...des hab i eh erzählt das $i$ mit dreizehn mir das abtrainiert hab, es war eher so ein latenter Prozess, aber es war, $i$ bin dann schon öfter auf mein rollendes $R$ angesprochen worden und dann hab i mir gedocht i leb eh in Kärnten oder so, dass kann i mia ja leicht abtrainieren mach mas und irgendwie äh bis auf meinen Namen kann, könnt man mi jetzt im Gespräch jetzt garnet auf mei Herkunft äh in Verbindung setzen [...] weil man irgendwie subtil immer damit in Verbindung gebracht wird, vor allem, wenn man's noch raushört" (Interview A, Zeile 485f.).

Abtrainieren kann nicht „latent“ verlaufen; es ist stets bewusste Arbeit, hier: die Arbeit an der eigenen Sprache und am eigenen Selbst - die Selbstoptimierung. Die Initialzündung war die Auffälligkeit des rollenden Rs, die Tatsache, dass sie darauf angesprochen wurde. In Kärnten fallen rollende Rs auf, sie sorgen für Reibung. Doch Oberflächen müssen glatt sein (Alvir 2015: 138), Hybridität ist nur schwer erträglich. Eindeutigkeit wird erwartet; sie wird erfragt. In allen vier Interviews beschreiben die InterviewpartnerInnen, dass es andere sind, die ihnen eine Entscheidung abverlangen: ,Was bist Du? Entweder - oder! Entscheide Dich!' Oder die Eindeutigkeit wird durch andere (typischerweise in Abhängigkeit vom sozialen Kontext) geschaffen: ,Du bist ÖsterreicherIn, du bist BosnierIn, Du bist SerbIn/KroatIn/MuslimIn!'. Die Zugehörigkeit wird verdinglicht und als unverrückbar definiert. Ähnlich den ProtagonistInnen in den Romanen sind auch unsere InterviewpartnerInnen in einem „Dazwischen" verortet. Diese Positionierung kommt in allen vier von uns zur Analyse herangezogenen Interviews zum Ausdruck. Und wie die Romanfiguren sehen auch sie sich mit Wirklichkeiten konfrontiert, die ihnen entweder eine eindeutige Entscheidung abverlangen (vgl. z.B. Alvir 2015: 40; Gugić 2015: 61), oder aber die Entscheidung für sie fällen (vgl. z.B. Dabić 2017: 6o; Alvir 2015: 224). Die Sprecherin der hier zitierten Passagen begegnet den gesellschaftlichen Erwartungen, indem sie die Oberfläche ihrer Sprache glättet, indem 
sie das, was sie als potenziell Außenstehende outet, verschwinden lässt; sie möchte mit ihrer Herkunft nicht in Verbindung gebracht werden und macht aus diesem Grund einen Teil ihrer Selbst unsichtbar - Selbstoptimierung ist das Ziel. Geglättet wird aber nicht nur die Oberfläche der Sprache, geglättet werden auch potenzielle Reibungspunkte im „Dazwischen“. Die Verortung „Zwischen zwei, drei Stühlen“ wird, wie eben dargelegt wurde, entproblematisiert und, einer Verwertungslogik folgend, als eine profitable Position beschrieben.

Die Analyse der Passagen liefert auch Hinweise darauf, wie sich diese Positionierung auf das Selbstbild der Sprecherin auswirkt: Trotz der Glättungen im (problematischen) Inner-[Außer-]Halb verbleibend, greift sie bei der Beschreibung ihrer selbst auf Stereotype zurück. Stereotypisch wird in der Regel aber nur Fremdes beschrieben. Lässt das Nachdenken über die eigenen Wurzeln die Sprecherin sich selbst fremd werden? Diese Frage kann an dieser Stelle noch nicht beantwortet werden, die Fallstruktur ist noch nicht geschlossen. Sie lässt uns aber an eine Passage aus Alvirs Roman denken. Hier konstatiert Lara an der Schnittstelle ihrer Rollen als Schönheitsexpertin und Familiendetektivin: „Bloß sollte eins sich nicht zu viel mit den eigenen Genen beschäftigen, und schnell kommt man auf die Idee, etwas Besonderes zu sein. Wer nach seinen Wurzeln fragt, begreift sich als Frisur" (Alvir 2015: 40).

Wir wollen im Folgenden nun einen Blick darauf werfen, wie die ProtagonistInnen der untersuchten Romane sowie die anderen InterviewpartnerInnen mit der Verortung im „Dazwischen“ und den sie umgebenden Erwartungen umgehen.

\section{Kompensationsstrategien}

Die Reaktionen der Romanfiguren auf eine verdinglichte Welt, in der ihr Menschsein keinen Platz mehr zu haben scheint, präsentieren sich auf unterschiedliche Art und Weise. Wir haben einige typische Reaktionsmomente herausgegriffen und mit dem Interviewmaterial verglichen. Vorausgeschickt sei dieser Gegenüberstellung, dass wir uns der unterschiedlichen Räume bewusst sind, die in diesem Vergleich einander gegenübergestellt werden (Bourdieu 1999, Bourdieu 2011): Der fiktionale Raum der Werke und der gesellschaftliche Raum - das soziale Feld, in dem sich die InterviewpartnerInnen bewegen. Die Gesetze, denen der fiktionale Raum der Werke gehorcht, sind erfunden wie auch die RomanprotagonistInnen selbst; dennoch ist die Romanwirklichkeit strukturhomologisch ver- wandt mit der Positionierung und daher auch mit der Wahrnehmung und Perspektive der sozialen Welt der jeweiligen Autorin. Insofern stehen sich literarisches und soziales Feld in unserem Vergleich gegenüber. Um auf die strukturellen Unterschiede der jeweiligen Felder genauer eingehen zu können, bedürfte es einer separaten Analyse, die u.a. innerhalb des literarischen Feldes genauer differenzieren müsste. Unser Schwerpunkt liegt in der vorliegenden Untersuchung jedoch auf der gesellschaftlichen Bilanz der Verdinglichung, die wir sowohl aus der einen, der sozialen/gesellschaftlichen, als auch der anderen, der fiktionalen Wirklichkeit, ziehen und hier zur Debatte stellen. Die literarischen Publikationen sind bereits ein Verweis darauf, dass die Stimmen der Autorinnen, im Gegensatz zu jenen der InterviewpartnerInnen, eine andere Öffentlichkeit haben und eine andere Wertschätzung (künstlerisch/ kulturell) genießen. Damit geht auch einher, dass die Autorinnen in anderer Form gesellschaftliche Kritik äußern können/dürfen, selbst wenn diesbezüglich hierarchische Strukturen innerhalb des literarischen Feldes zu bedenken sind. Jedenfalls verfügen die Autorinnen über eine Handlungs- und Protestfähigkeit, d.h. eine Form der Autonomie und Beweglichkeit, über die ihre ProtagonistInnen oder unsere InterviewpartnerInnen nicht notwendigerweise verfügen; das Erzählen selbst und die Beherrschung von Sprache werden für die Autorinnen zum einen wichtige Identifizierungs-, zum anderen aber auch Reibungsinstrumente, die der Gesellschaft einen Spiegel vorhalten. Diesen Blick in den Spiegel halten wir für lohnend, ohne dabei einen direkten Vergleich weder der Verortung noch der individuellen Lebenswirklichkeiten der jeweiligen SprecherInnnen ziehen zu wollen.

Ein wichtiges Reaktionsmoment auf die verdinglichte Welt scheint etwa der Versuch zu sein, sich zu assimilieren (Gugić 2015: 180), sich glatt (Alvir 2015: 138) oder schwerelos zu machen (vgl. dazu die Leitmetapher der/s Astronautin/en in Gugić 2015). Das gezielte Abtrainieren des Akzents, genauer: des rollenden Rs kann als ein solcher Versuch der Assimilierung, der vollkommenen Eingliederung, gelesen werden; womöglich verursacht durch eine durch Umwandlung von Fremd- in Selbstzwänge produzierte Scham (Elias 1997): „...Mein Leben als junges Jugo-Kind. Ich habe mich dafür geschämt, dass ich mein $\mathrm{R}$ rolle (F: Dass Du Dein R rollst? IB: Ja. (2) Fand ich nicht so toll, weil sie sofort wussten, dass ich von irgendwo kam, was nicht Österreich ist." (Interview B, Zeile 235ff.) - so eine zweite Interviewpartnerin mit einer Aussage, die 
sowohl inhaltlich als auch formal den Äußerungen der ersten Gesprächspartnerin frappierend ähnelt.

Die Angst davor, den Ansprüchen nicht gerecht werden zu können, kann aber auch zu einem vollkommenen Verstummen oder Erstarren führen, zu dem Versuch, sich unsichtbar zu machen (Dabić 2017: 50): „... das erste halbe Jahr habe ich überhaupt (.) gar nicht gesprochen“ (Interview C, Zeile 73) - so eine unserer GesprächspartnerInnen. Dieses Moment des Verstummens findet sich auch in den Romanen wieder. Das „Lauskind“, Laras kindliches Alter Ego, öffnet nach seiner Flucht von Bosnien nach Österreich erst nach zwei, drei Jahren wieder den Mund, nachdem es Deutsch erlernt, d.h. also eine sprachliche Entsprechung zu dem System, in das es eingegliedert wurde, gefunden hatte (Alvir 2015: 13). Nora, der Dolmetscherin, wird abverlangt, zugunsten des Systems nicht in Erscheinung zu treten. Unsichtbar muss sie sein: Sie ist „die Hörende und Verstehende“, die so viel spricht „wie die beiden anderen zusammen, die aber von sich aus nichts sagen [darf]“ (Dabić 2017: 50). Und die Sprache von Alen - dem Schriftsteller unter Gugićs AstronautInnen - geht zwischen den Manuskriptseiten, die er unentwegt zwischen zwei Sprachen hin- und herübersetzt, niemals aber veröffentlicht, verloren (Gugić 2015: 13). Das Gefühl der Unvollständigkeit, das ihn beim Aufspüren unzähliger „Fehler, Ungereimtheiten, in jeder Sprache andere" (Gugić 2015: 13), verfolgt, ist wie eine offene Wunde. Wie das Gefühl, weder der einen Front noch der anderen gerecht zu werden. Vielleicht aus diesem Gefühl des ,Niemals-tatsächlich-entsprechen-Könnens' heraus kultiviert Alen einen würdigen Auftritt in der Öffentlichkeit: Im Anzug fährt er Taxi - „als wäre er ein Privatchauffeur, der gleiche Anzug in drei Ausführungen, dazu drei identische, passende Hemden" (Gugić 2015: 13). So als habe er dreifach seine Schuldigkeit zu tun, sein ,Unvermögen' zu kompensieren. Spuren eines solchen übersteigerten Ausgleichens finden sich auch im Interviewmaterial wieder. Am deutlichsten vielleicht bei der Sprache selbst - so sprechen etwa drei der vier InterviewpartnerInnen mit einem sehr ausgeprägten Dialekt.

Olja Alvir lässt hingegen ihre Protagonistin schwanken zwischen ihrem Eifer, der Norm, u.a. dem Anspruch einer oberflächlichen Makellosigkeit, nachzukommen (Alvir 2015: 138), und dem Verlangen, ihrem inneren Überdruck (der „Hitze im Inneren“), dem vernachlässigten Selbst, über die Verletzung und ,Öffnung ihrer Oberfläche nachzugeben: Sie ritzt ihre Haut, damit die Abweichungen zwischen „Angstmaschinen-Ich und Normal-Ich langsam wieder kongruent werden“ (Alvir 2015: 197). Scheinbar übernimmt sie so die Kontrolle über ihren Körper, den Susan Bordo in ihrem Essay „Anorexia nervosa“ als vielleicht „one of the few available arenas of control we have left in the twentieth century" bezeichnet (Bordo 1997: 228). Tatsächlich birgt der Akt der Kontrollübernahme, der Herrschaft über das Selbst, gleichzeitig das Verlustmoment der Selbstbeherrschung. Dazu ließe sich weiter in Anlehnung an Susan Bordo argumentieren: „Paradoxically - and often tragically - these pathologies of female protest (...) [Susan Bordo nimmt hier Bezug auf: Bulimie, Anorexie und Agoraphobie] actually function as if in collusion with the cultural conditions that produced them." (Bordo 1997: 241) Eine Interviewpartnerin versucht die Kontrolle über die Kommunikation und ihre Herkunftsbestimmung in Situationen, in denen sie „blöd“ danach gefragt wird, an sich zu reißen: „Ja, vor ein paar Jahren hab' ich damit angefangen zu sagen, ich komm' aus Jugoslawien. (.) Aus einem Land, das es nicht mehr gibt. Warum? Um die Leute zu verwirren“" (Interview D, Zeile 255ff.). Dieser Verweis auf einen nicht mehr existenten Ort könnte darauf hinweisen, dass die Sprecherin sich letztlich als, herkunftslos' betrachtet. In jedem Fall sorgt sie aber hier für zusätzliche Reibung, sie provoziert. Das Muster einer humoristischen Provokation reproduziert sich in diesem Interview immer wieder. Auch diese Gesprächspartnerin greift beispielsweise bei der Beschreibung der kulturellen Einflüsse auf Stereotype zurück, doch sie tut es auf eine sehr humorvolle Art, was darauf schließen lässt, dass sie gleichzeitig auch darüber reflektiert: „(...) die Österreicherkinder haben immer nur gesagt: ,wir geh'n Kaffee trinken und und einen Kuchen essen bei der Oma und bei uns war's halt Schnaps und ein Spanferkel“ (Interview D, Zeile 297ff.).

Eine andere Reaktion auf die Verdinglichung ist die Resignation aus Überforderung, wie sie sich etwa in der von Gugić beschriebenen Szene des Selbstmords (hier finden sich auch Parallelen zum Verstummen oder Erstarren) aufdrängt. Darko, ein weiterer Astronaut aus Gugićs Roman, kann etwa in der Flut von Nachrichten, Inschriften und Signalen der Stadt keinen Sinn mehr erfassen und zieht sich aus der funktionalen Welt zurück: „Eine Abfolge, die immer mehr durcheinandergerät, bis der Text in ein Wirrwarr aus Buchstaben zerfällt, in Nullen und Einsen, schließlich fällt der eine Monitor aus, Signal Error, während die Bilder auf dem anderen erst nur einen Tick zu schnell, dann in manischer Abfolge weiterlaufen, vor meinen Augen zu flimmern beginnen, bis ich wegsehen muss" (Gugić 2015: 115). 
Und ein dritter (Niko) entzieht sich in unbeobachteten Momenten dem Perfektionismus, den ihm sein Umfeld abverlangt. Dann sucht er die Schwerelosigkeit in der gedämpften Unterwasserwelt der Badewanne oder des Schwimmbads, macht sich taub gegen die mahnende Stimme der Welt der Vorschriften. Er weicht seinen Körper auf und löst sich aus seiner Umwelt (Gugić 2015: 142f.) - zumindest auf Zeit. Nora, die Dolmetscherin, wiederum versinkt mitunter in dem Niemandsland zwischen zwei GesprächspartnerInnen. In der Vermittlung gefangen, läuft sie Gefahr, sich selbst zu verlieren: „Und ihre Stimme hörte sich fremd an" (Dabić 2017: 38). Ihre Verortung ist in Schwebe, sie ist ihrer eigenen Schwerkraft beraubt.

Auch die Analyse unserer narrativen Interviews legt solche Momente der Resignation offen: „außerirdisch“ (Interview D, Zeile 364) - und damit eben auch in der Schwebe - fühlt sich eine Interviewpartnerin, wenn sie über ihre Zugehörigkeit nachdenkt; „mir ist des egal“ (Interview B, Zeile 19) - ist die Reaktion eines anderen auf die Versuche seines Umfelds, ihn mit eindeutigen Identitäten zu etikettieren. ${ }^{\text {"1 }}$ Andere dagegen übernehmen die Etiketten ihrer Umgebung und verwenden sie für ihre Selbstbeschreibung: „Ich als Ausländerkind“, "Jugo-Kind“, „Drittstaatenangehöriger“ etc.; eine Art der Resignation ist aber auch die Reduktion des (Da-) Seins auf den formalen Status: Der Pass sagt, wer ich bin - so in etwa ein Interviewter.

Beides zusammen, die Übernahme der Etikette und die Reduktion des Seins auf den formalen Status, kommen prägnant in Dabićs Roman zum Ausdruck. In der eingangs zitierten Passage beschreibt Dabić, dass sich das Warten auf den Asylbescheid in jede Faser des Körpers der Wartenden einschreibt. Der eingetroffene Asylbescheid ändert den Aggregatszustand, er bescheinigt das Dasein, die greifbare Existenz. Auch in der Selbstwahrnehmung wird das Sein auf die Asyl-

11 „Ajo und wenn i donn unten bin, also früher war's immer so ähm, dass wi i dann in Bosnien war, da hom dann die Meisten irgendwie, vor allem die die die in meinem Alter war'n, di hom imma g'sogt, dass i der Österreicher bin. (2) Aber die, die äh die Verwandschaft in Österreich, die hot imma g'sogt, dass i ka Österreicher bin, sondern a Bosnier. (2) Oder vielleicht net ganz Bosnier, sondern die hom ja ähm g'sogt, dass i a Sss..erbe bin (.), also wegen da wegen da wegen da ethnischen Zugehörigkeit- Weil da gibt's ja des (2) da hom sie a paar Schwierigkeiten. (2) Das haßt die Meist ... also die aus Bosnien sind, die serbisch-orthodox sind, die nennen sich ja selber Serben (.) und Bosnier, die wirklich Bosnier sind, (.) die sind dann meistens Muslime (.) meistens. Aber mir is des egal“" (Interview B, Zeile 114ff.). entscheidung reduziert: Jeder Individualität beraubt, bezeichnen sich jene, die nur lange genug gewartet haben, als „Positiwschiza“ oder „Negatiwschiza“ (Dabić 2017: 60). Reibungslos soll es laufen und deshalb zeichnet das System - anders als es Dabić in ihrem Roman beabsichtigt - keine Menschen, sondern „Abziehbilder“ (Biller 2017).

\section{Conclusio}

Die eben skizzierte Untersuchung ist als ein work in progress zu verstehen. Sie stellt eine erste analytische Annäherung an die narrativen Interviews und die sich darin reproduzierenden Selbstbilder dar und eröffnet einen Dialog zwischen Interviewmaterial und literarischen Werken.

Wenn wir nun aber an dieser Stelle ein Zwischenresümee ziehen wollen, so können wir festhalten, dass sich sowohl in den literarischen Werken der von uns behandelten Autorinnen - Gugić, Alvir und Dabić - als auch in dem analysierten Interviewmaterial die Konfrontation mit erstarrten und enthumanisierten Wirklichkeiten einerseits und die Positionierung in einem Inner-[Außer-]Halb andererseits wiederfindet. Auch hinsichtlich der Reaktionen der InterviewpartnerInnen und ProtagonistInnen auf die verdinglichte Welt und ihre eigene Verortung im „Dazwischen“ lassen sich, wie wir in unserem letzten Teil darzulegen versucht haben, zahlreiche Parallelen identifizieren:

Glätten (durch Selbstoptimierung und, wie vor allem im Fall A herausgearbeitet, durch die Unterwerfung unter die Logiken des Konsums, der Verwertung und des Profits), Überkompensieren, Verstummen, Erstarren oder Resignieren, oder aber Kontrollübernahme und gezielte Erzeugung von Reibung, indem man etwa Verwirrung stiftet oder sich gezielt über gesellschaftliche Tabus hinwegsetzt.

Sensibilisiert durch die Analyse möchten wir an dieser Stelle nun aber auch den eingangs als Antithese zu Dabićs Romantitel Reibungsverluste abgeleiteten Begriff des Reibungsgewinns noch einmal grundsätzlich überdenken. Indem wir uns mit dieser Formulierung in eine Logik der sich ausschließenden Gegensätze einreihen, reproduzieren wir einen Diskurs, der nach Maßstäben eines „Entweder-oder“ operiert. Darüber hinaus birgt die Identifikation von Reibungsgewinnen auch die Gefahr, einer VerWERTungslogik (damit auch einer Form des Materialismus) aufzusitzen. Die Auseinandersetzung mit dem Eigenen und damit dem mutmaßlich Anderen bedeutet immer auch, Konflikte und 
Veränderungen zuzulassen, ohne die daraus entstehenden neuen Wege und Entwicklungen wieder in Formen des Gewinns oder des Verlustes zu pressen. Daher wollen wir der in der Gesellschaft identifizierten Entmenschlichung vielmehr mit dem Begriff der Reibung begegnen. Reibung taucht dort auf, wo Begegnung und Kommunikation stattfinden. Sie erfüllt gleichsam den Begegnungsraum des „Dazwischen“ mit (Reibungs-) Wärme und trägt zur Überwindung von Grenzen und dualistischen Denkmustern bei.

Die spezielle Situation einer Verortung zwischen zwei oder drei nationalen Kategorien, wie wir sie hier schwerpunktmäßig diskutiert haben, legt unseres Erachtens den Finger auf zwei zusammenhängende Tendenzen in unserer Gesellschaft, die selbst durch unzählige, viel subtilere und schwerer identifizierbare Kriterien des Ein- und Ausschlusses festzementiert sind: eben die Tendenzen der Verdinglichung - der Erstarrung -, und der Entmenschlichung.

IHNEN zu begegnen stellt eine der großen Herausforderungen unserer Gesellschaft dar und bedarf unseres Erachtens des Zulassens von Reibung, von Konflikt, und einer Bereitschaft, das Bequeme, das Zweifelsfreie aufzugeben - ohne dabei die eigene Stimme zu verlieren oder anderen die ihre zu nehmen. Das hierbei freigesetzte Potenzial - die (Reibungs-)Wärme, der Impuls (engl. momentum) oder das von Homi Bhabha beschriebene Darüber-Hinaus - könnte der Bedeutung des dem Lateinischen entlehnten integrare (erneuern, ergänzen, geistig auffrischen) in der Gesellschaft tatsächlich Platz einräumen. Eine Auffassung des Integrationsbegriffs, die a priori von der Vielheit und Veränderlichkeit von Identitäten ausgeht, würde auch innerhalb der Systeme mehr Platz für das menschliche Bedürfnis nach Lebendigkeit und Toleranz schaffen.

\section{Literatur}

Alvir, O. (2015): Kein Meer. Wien: Zaglossus.

Anthias, F. (2001): New Hybridities, Old Concepts: The Limits of ,Culture. Ethnic and Racial Studies, 24 (4), 619-41.

Anthias, F. (2002): Where Do I Belong? Narrating Collective Identity and Translocational Positionality. Ethnicities, 2 (4), 491-514.

Augé, M. (1992): Non-lieux. Paris: Seuil.

Berger, P. L./Luckmann, T. (1969): Die gesellschaftliche Konstruktion der Wirklichkeit. Eine Theorie der Wissenssoziologie. Frankfurt/M.: Fischer.
Berger, P. L./Pullberg, S. (1965): Verdinglichung und die soziologische Kritik des Bewusstseins. Soziale Welt, 16 (2), 97-112.

Bhabha, H. K. (200o): Die Verortung der Kultur. Tübingen: Stauffenburg.

Biller, H. (2017): Mascha Dabić: ,Ich will Menschen, keine Abziehbilder zeigen. Der Standard. Online: https:// derstandard.at/2000055183353/Mascha-Dabic-Ich-willMenschen-keine-Abziehbilder-zeigen[17.04.2018].

Bordo, S. (1997): Anorexia Nervosa. Psychopathology as the Crystallization of Culture. In: Counihan, C./Esterik, P. V. (Hg.): Food and Culture. A Reader, New York: Psychology Press, 226-250.

Bourdieu, P. (1999): Die Regeln der Kunst. Genese und Struktur des literarischen Feldes. Frankfurt/M.: Suhrkamp.

Bourdieu, P. (2011): Das literarische Feld. In: Bourdieu, P.: Kunst und Kultur. Kunst und künstlerisches Feld. Schriften zur Kultursoziologie. Band 4. Konstanz: UVK, 309-337.

Cooley, Ch. H. (1964): Human Nature and the Social Order. New York: Scribner's.

Dabić, M. (2017): Reibungsverluste. Wien: Edition Atelier.

Elias, N. (1997): Über den Prozess der Zivilisation. Soziogenetische und physiogenetische Untersuchungen, Band 2: Wandlungen der Gesellschaft. Entwurf zu einer Theorie der Zivilisation. Frankfurt/M.: Suhrkamp.

Frühwirth, A./Mijić, A. (2016): An die Grenzen des Selbst. Identität \& Diaspora. Beitrag zur 4. Jahrestagung der Migrations- und Integrationsforschung in Österreich der Österreichischen Akademie der Wissenschaften und der Universität Wien 2016. Manuskript.

Frühwirth, A./Mijić, A. (2017): F(r)iktionen. Eine Sozialphysik der Reibungslosigkeit. Psychologie \& Gesellschaftskritik, 41 (1), 29-48.

Gugić, S. (2015): Astronauten. München: C.H.Beck.

Kalthoff, H. (2008): Einleitung. In: Hirschauer, S./Kalthoff, H./Lindemann, G. (Hg): Theoretische Empirie. Zur Relevanz qualitativer Forschung. Frankfurt/M.: Suhrkamp, $8-32$.

Levitt, P. (2009): Roots and Routes. Understanding the Lives of the Second Generation Transnationally. Journal of Ethnic and Migration Studies, 35 (7), 1225-1242.

Loch, U./Rosenthal, G. (2002): Das narrative Interview. In: Schaeffer, D./Müller-Mundt, G. (Hg.): Qualitative Gesundheits- und Pflegeforschung. Bern et al.: Huber, 221232.

Luckmann, T./Berger P.L. (1980): Soziale Mobilität und persönliche Identität. In: Luckmann, T.: Lebenswelt und Gesellschaft. Grundstrukturen und geschichtliche Wandlungen. Paderborn: Schöningh, 142-160.

Mead, G.H. (1934): Mind, Self and Society. Chicago: University of Chicago Press.

Oevermann, U. (1990): Exemplarische Analyse eines Gedichts von Rudolf Alexander Schröder mit dem Verfahren der objektiven Hermeneutik. Kulturanalysen. Zeit- 
schrift für Tiefenhermeneutik und Sozialisationstheorie, 2 (3), 244-260.

Oevermann, U. (2002): Klinische Soziologie auf der Basis der Methodologie der objektiven Hermeneutik: Manifest der objektiven hermeneutischen Sozialforschung. Online: https://www.ihsk.de/publikationen/Ulrich_OevermannManifest_der_objektiv_hermeneutischen_Sozialforschung.pdf [27.04.2018].

Oevermann, U./Allert, T./Konau, E./Krambeck, J. (1979): Die Methodologie der objektiven Hermeneutik und ihre allgemeine forschungslogische Bedeutung in den Sozialwissenschaften. In: Soeffner, H.-G. (Hg.): Interpretative Verfahren in den Sozial- und Textwissenschaften. Stuttgart: Metzler, 352-434.

Park, R. E. (1928): Human Migration and the Marginal Man. American Journal of Sociology, 33 (6), 881-893.

Park, R. E. (1950): Race and Culture. Glencoe, Ill: Free Press.

Said, E. W. (2002): Reflections on Exile and Other Essays. Cambridge: Harvard University Press.

Schütz, A. (1972): Der Fremde. In: Gesammelte Aufsätze, Band 2: Studien zur soziologischen Theorie. Den Haag: Nijhoff, 53-69.

Schütz, A./Luckmann, T. (2003): Strukturen der Lebenswelt. Konstanz: UVK.

Somerville, K. (2008): Transnational Belonging among Second Generation Youth. Identity in a Globalized World. Journal of Social Science, Special Issue (10), 23-33.

Waters, M.C./Levitt, P. (2002): The Changing Face of Home. The Transnational Lives of the Second Generation. New York: Russell Sage Foundation.

Winker, G./Degele, N. (2010): Intersektionalität. Zur Analyse sozialer Ungleichheiten. Bielefeld: Transcript. 